\title{
ILRs- Implantable Loop Recorders, Insertion Guidance and After Care Precautions- A Review and Reflection of the Literature and Our Experience
}

\author{
Kathleen Fasing ${ }^{1 *}$ and Suzanne Benloucif ${ }^{2}$ \\ ${ }^{1}$ University of Michigan Cardiovascular Center, East Medical Center Drive Ann Arbor, USA \\ ${ }^{2}$ University of Michigan Cardiovascular Department Electrophysiology Department, Adjunct Department of Nursing Ann Arbor, \\ USA
}

*Corresponding author: Kathleen Fasing, University of Michigan Cardiovascular Center 2A- 5869, 1500 East Medical Center Drive

Ann Arbor, MI 48109-5869, USA

\section{ARTICLE INFO}

Received: 幽 June 15, 2021

Published: 豐 June 21, 2021

\section{ABSTRACT}

Citation: Kathleen Fasing, Suzanne Benloucif. ILRs- Implantable Loop Recorders, Insertion Guidance and After Care Precautions- A Review and Reflection of the Literature and Our Experience. Biomed J Sci \& Tech Res 36(4)-2021. BJSTR. MS.ID.005887.

\section{Indications for Implanted Loop Recorders}

In the cardiology and electrophysiology clinic there are several diagnoses which challenge the provider to determine the specific arrhythmia. These challenges include consideration of the best way to capture tracings or evidence of the arrhythmia. Patient complaints may include a fluttering sensation and palpitations in one's chest, a history of cryptogenic stroke, passing out, a history of atrial fibrillation which occurs very infrequently, and history of seizure with suspected arrhythmia trigger [1]. The provider determines the best monitoring device which can capture the underlying rhythm for such complaints. Monitoring devices are externally worn devices which detect changes in heart rhythms [2]. There are several monitors which have specialized indications. There are two week monitors which are placed externally and data is sent for interpretation (such as a brand called the Ziopatch [3].

These monitors can be minimally affected by showering or a plastic barrier used to protect the monitor from water [4]. Another type of monitor is a two-day monitor which is ordered when a patient has a frequently occurring arrhythmia and the overall percentage of the arrhythmia is needed. These monitors are called Holter monitors and are excellent in giving an overall ventricular arrhythmia burden or premature ventricular burden [5]. There are also externally worn event monitors designed to be a real time system to directly alert the provider of an adverse cardiac event. This type of monitoring system relies upon focused and intense monitoring which gives timely and current communication to the provider, rather than a historic encapsulation of the heart rhythm events. Such real time event monitors are offered by such companies, such as the Biotel monitor [3].

Unfortunately, some arrhythmias occur so infrequently that an externally worn device with a preset time such as a few days or 2-4 weeks may not capture the arrhythmia. There are special circumstances in which a long-term monitoring device is indicated. Such a device is a loop recorder (implanted loop recorder- ILR) that is implanted subcutaneously and transmits the arrhythmias to a remote monitor. These devices capture the abnormal heart rhythm and transmit the data which may be uploaded and evaluated later. Dangerous, but infrequently occurring arrhythmias such as nonsustained ventricular tachycardia and prolonged pauses or short episodes of asystole which later require pacemaker insertion have become indications for ILR as the ILRs are able to capture these infrequently occurring arrhythmias given the ongoing monitoring capability [3].

Loop recorders are implanted devices which can track any arrhythmia which the patient experiences. These devices last over 
a preset length of time determined by the device's internal battery life with the average LINQ battery life estimated at three years [6], Indications for loop recorders include syncope, cryptogenic stroke to assess for atrial fibrillation, palpitations and resultant symptoms, and a need to gain an overall atrial fibrillation burden [7]. The ILR devices provide ongoing monitoring and can capture any arrhythmia over several years. They function in a retrospective fashion and give the provider a mirror of trends in arrhythmia or unusual changes in the heart rhythm. Coupled with a careful journal of patient complaints, they provide vital information from which the provider makes the diagnosis and treatment plan. Indications for ILR devices are outlined by the Heart Rhythm Society. If an indication for an ILR implant is verified one can then plan and expedite the ILR implant procedure.

\section{FDA Approval for Small-Implanted Loop Recorders}

The process of monitoring one's arrhythmias became much easier in February 2014 with the release of the LINQ monitor from the Medtronic ${ }^{\mathrm{TM}}$ corporation, the smallest monitoring device available. This device is a subcutaneously placed monitor which is one third the size of a triple A battery, $1.2 \mathrm{cc}$ and transmits data via remote monitoring [6]. It then quickly became an easy way to determine abnormal arrhythmias and possible causes of strokes, which may have eluded periodic external monitoring techniques in the past [8]. The following is an overview of the use of the LINQ monitor, expected outcomes, unusual ILR complications and trouble-shooting tips for the provider.

\section{Evolvement of Procedure Room; Holding Room Insertions of ILRs}

With the introduction of the Medtronic LINQ ILR and its lowprofile size, weight, and subcutaneous injection delivery system came great excitement for such an easy delivery system of such an advanced device. Many facilities started performing the insertion of the ILRs in other treatment areas, rather than the cardiac catheterization labs and electrophysiology labs. The ease of the insertion of the device led to these devices being implanted with advanced professional staff such as physician assistants and nurse practitioners in procedure rooms and holding bays. Following this change in practice from a conventional lab setting, there were several studies to analyze potential side effects and the efficacy of this new practice. Four studies in particular show a low likelihood for adverse events and review some learned pearls of knowledge for the insertion and care of these devices. The four cases involving LINQ studies and studies of in office, holding room, procedure room and advance practice profession placement of the devices are outlined in the literature review.

\section{Potential Side Effect Profile}

In our practice of a 1000 bed tertiary care facility and EP lab which implants approximately 250 loop recorder devices per year [9], we have a very low side effect profile, much as is seen nationally. As in previous loop recorder studies [10] our side effect profile includes localized bleeding (small hematoma), localized skin changes (irritation and or rash) and localized infection or mild pain at the insertion pocket site [11]. These side effects are limited to a less than $1-2 \%$ chance of occurrence and are easily managed with short term compression, skin care and acetaminophen or ibuprofen. Of note, we are particularly interested in 4-5 scenarios which have presented in the last 3-4 years with patients complaining of recurrent and very intense pain at the device site requiring prescribed narcotic medications, ice and compression of the area, and in two cases, removal of the loop recorder. Of note, two of the instances include devices which were placed at outside hospitals and our service simply saw the patient due to change in living locations or transferring from another region of the country.

\section{Typical Implanted Loop Recorder Practices}

We first reviewed our typical placement of loop recorders and the precautions we take with every placement of an internal cardiac monitoring device. In analyzing possible pain or unusual cases involving a loop recorder one must first review internal policies on placements of such devices as well as perform a literature search. By analyzing possible sources of complications or untoward events one may avoid such events and learn from literature on loop recorder devices which give tips on avoiding untoward events. At our tertiary care- facility the method of implant either utilizes local anesthesia or a combination of local anesthesia with bupivacaine/ lidocaine/epinephrine placed subcutaneous and sedation with mild analgesic if necessary. All loop recorder implants are done within the EP laboratory. The location in the chest of the implant is left chest, 4 th intercostal space, $2-3 \mathrm{~cm}$ from the sternum. A prep resistant marker is used to identify the insertion site. An informed consent is gained from the patient prior to entering the lab. The patient's insertion site is prepped with Chlorhexidine and draped using sterile technique.

Five milliliters of local anesthesia (as above) is injected into the subcutaneous space. An incision is then made utilizing the angled blade provided in the LINQ-tm insertion kit. The monitoring device is inserted into the subcutaneous space parallel to the skin utilizing the insertion tool. The tool is then turned 180 degrees to create the pocket. While firmly holding the insertion tool against the skin, the device is injected into the subcutaneous tissue parallel to the skin. The device is interrogated to assure proper sensing. The device is programed based upon the diagnosis. Pressure is then 
held for 5 minutes to ensure hemostasis [6]. Glue is applied to the skin, followed by steri-strips and a telfa pad $[6,12]$. Some of our physicians prescribe antibiotics prior to the procedure and some do not, depending upon personal preference (Figure 1).

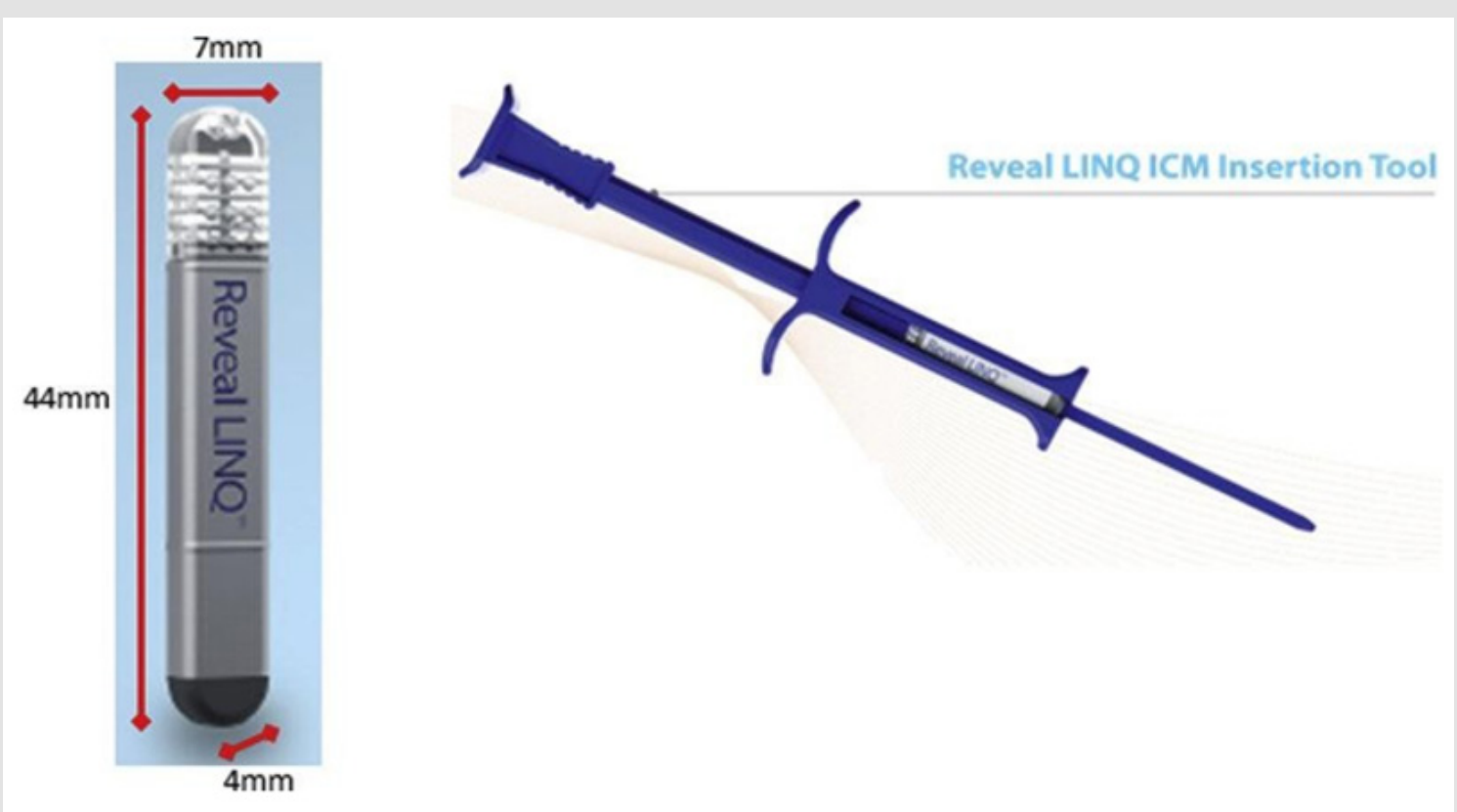

Figure 1: Picture from Medtronic advertising site.

\section{Picture from Medtronic Advertising Site}

After completion of the procedure care includes removing the dressing in 48 hours. Leave the steri-strips in place until they start to peel off. Patient education is completed in the recovery area prior to patient being discharged from the area. A wound check is scheduled and maintained in 7-10 days.

\section{Size of Devices}

(Figure 2).

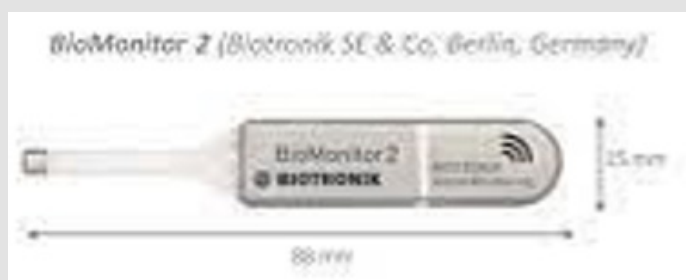

Rewsol XT iMedtronic, Minneopoles, USA

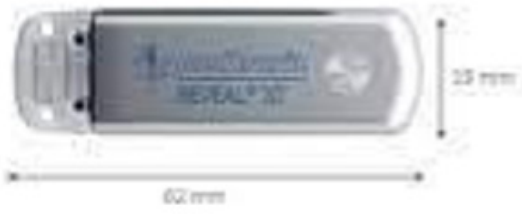

\section{Rereal LWQ (hestronic, Minnesponts, USA)}

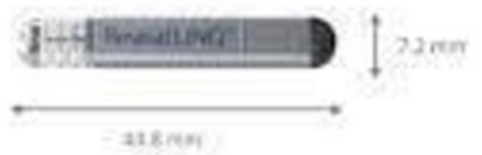

Confinm Rr -jOU (St Jude Mectical. Minncsoto. USA)

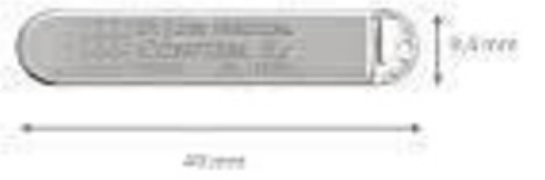

Figure 2.

\section{Literature Review}

This literature review examined advantages of an ILR, evolving ILR technology, possible skin changes or rashes, increased pain at the insertion site, migration of the loop recorder and dislodgement of the loop recorder. This search proved to be challenging as the LINQ loop recorder insertion is associated with such a low complication rate and considered a smooth and relatively troublefree procedure [10]. The literature review revealed cases which included the term "pain" but did not directly relate to the pocket site pain. At our institution we have experienced 2-3 complaints which have experienced localized pain directly at the pocket insertion site. One article discussed a gentleman who initially experienced pocket site pain which improved but later developed chest and 
jaw pain. Such pain was initially felt to be anginal in nature and there was concern for an evolving ischemic cardiac event. He was evaluated for an acute coronary syndrome with a negative stress test. Within a day he was referred for an outpatient chest x-ray and was ultimately found to have a migrating loop recorder which was seen in the lower abdomen. The LINQ device was removed with an open surgical procedure [13].

There was an additional case of the LINQ migration involving a 78 year-old gentleman who had a LINQ loop recorder inserted with mild pain at the insertion site after the initial implant. The pain at the site slowly improved, but the patient developed pleuritic chest pain 35 days post ILR placement. The ILR had migrated to the left pleural space and was identified in this pleural space region on a computed tomography study (CT). The ILR was then removed with a video assisted thoracoscopic procedure utilizing a port and with forceps [14]. The final example of a LINQ loop recorder and pain is noted by Pocoro [15]. In this case of a 62-year-old gentleman with the placement of a loop recorder and prompt complaints of pain at the device site immediately post insertion of the device. He was discharged from the hospital despite his complaints of pain at the site and returned to the hospital on several occasions with similar complaints of pain at the insertion site of the loop recorder. The device was ultimately removed, but the device had been noted to be protruding from the insertion site. He was treated with pain medications, with little improvement. He ultimately required nerve blocks for pain management [15].

Another example of an ILR migration was a woman who underwent the ILR placement in the left parasternal location under local anesthesia. An assessment of the voltage signals followed with readjustment of the ILR device. Post procedure she experienced mild incisional pain and left breast discomfort which lasted several days. Her history included bilateral silicone breast implants. Her left breast pain resolved over time, but she noticed flattening of her left breast in comparison to the right breast. With follow up a computed tomography scan (CT) displayed the left breast implant rupture. The CT showed the ILR in close contact with the left breast rupture site [16].

The literature review gives a few key examples highlighting the need to fully investigate the complaints and possible etiologies of pain, with the patient who has such complaints after the placement of a LINQ loop recorder. There are other notations within the literature of rashes to the skin area of the insertion site and have later shown reactions to the metal and hypersensitivities to the metal (without actual allergy) which comprises the loop recorder [17]. Specific side effect profile noted by the Medtronic cooperation for the LINQ II loop recorder include device rejection phenomena (including local tissue reaction), device migration, infection and erosion through the skin [6].
Studies involving the side effect profile of the LINQ and insertion of the LINQ ILR are as follows. One study involved the University of Kansas Hospital and the Texas Cardiac Arrhythmia Institute in Austin Texas and compared conventional Medtronic Reveal loop recorder insertion to the subcutaneous injectable insertion of the ILR and side effect profiles [18]. They noted a higher infection rate in the implanted LINQ ILR as compared to the conventional implant of Reveal with surgical incision implant. The infection rate of the LINQ was $2-5 \%$ and occurred much earlier than the patients with the Reveal device. There was also a higher rate of wound dehiscence in this study. One can surmise these higher rates of infection and wound dehiscence may be due to the study being conducted within the year of the release of the LINQ device post FDA approval in 2014.

Rogers et. al. then conducted a study of in-office insertion of the LINQ device in 2017 and noted the most common side effect to be a site hematoma [19]. The study included 482 device -intra cardiac monitoring device (ICM) insertion with the LINQ-tm device and 244 of the 251 patients randomized to in office LINQ insertions and 227 of 231 patients randomized, in hospital insertion of the LINQ device. There were 4 insertion site complications with 2 complications in each group with a total of 4 events, with each group having one incision site hemorrhage and each group had one device dislocation due to erosion. There was no statistical difference in side effects post insertion between the in-hospital group and the in-office group [19].

There was another study evaluating the LINQ device implanted in an ambulatory setting by an advanced practice provider (APP) with outcomes showing no acute procedure complications. 125 patient ILR implants in the ambulatory setting inserted by APPs were assessed and subacute complications include two with one complication involving a suspected infection with erythema around the device site and subsequent empiric antibiotics provided. The other complication was local pain at the device site without infection one week post insertion which resulted in the device removal. The study demonstrated the low complication rate and efficacy of in office and APP placement of the LINQ device [20].

The final study also published in 2017 described low risk complication rates in the LINQ insertion within an electrophysiology lab as well as an outpatient procedure room within the LOOP study [21]. There were 1420 patients in the study and post implant pain was noted in 3 patients and an additional post implant pain complaint which resulted in the implant extraction. There was also noted minor bleeding or hematoma in 4 instances, superficial infection noted with 13 patients, and pocket erosion noted with 3 patients. There were a total of 18 devices which were removed and 9 of them were for pocket erosion, pain or infection and 9 were due to patient request with no physical abnormality [21]. 
Of interesting note, the patients with the higher body mass index (BMI) experienced more complications (which we have also observed within our practice and described to follow). The conclusion of the study is that the LINQ insertion is a very low risk procedure They noted a higher risk for those patients requiring repositioning of the device with a higher associated infection rate in those patients requiring repositioning outside of the conventional sterile electrophysiology laboratory setting [21]. The conclusion warns caregivers to be aware of the extra need for caution with patients with repositioning needs of the LINQ placed at the outside of the EP setting and a higher complication rate in either setting with those patients with a higher BMI [21].

\section{Our Typical Procedure Practice}

In implanting the ILR devices our facility has taken several precautions to ensure minimal risk and assure prompt healing from this minimally invasive procedure. ILRs are implanted in a sterile environment and using surgical sterile techniques. Care is given to insert the device subcutaneously, avoiding deep insertion with muscle or fat tissues. From our facility's practice we have experienced one event with a patient with morbid obesity in which the loop recorder was initially bothersome to the patient and ultimately needed forceps retrieval. Post procedural instructions are given to keep area clean and dry for the first one week. Instructions are given reviewing signs and symptoms of infection including the need to call if an evidence of redness or streaking at the site, any swelling which does not resolve in one to two days, any discharge from the site, any tissue changes showing redness, pulling apart of margins, or any unhealed margins which do not show closure of the insertion site.

\section{Picture of ILR tracing}

Tracing via Medtronic education site (Figure 3).

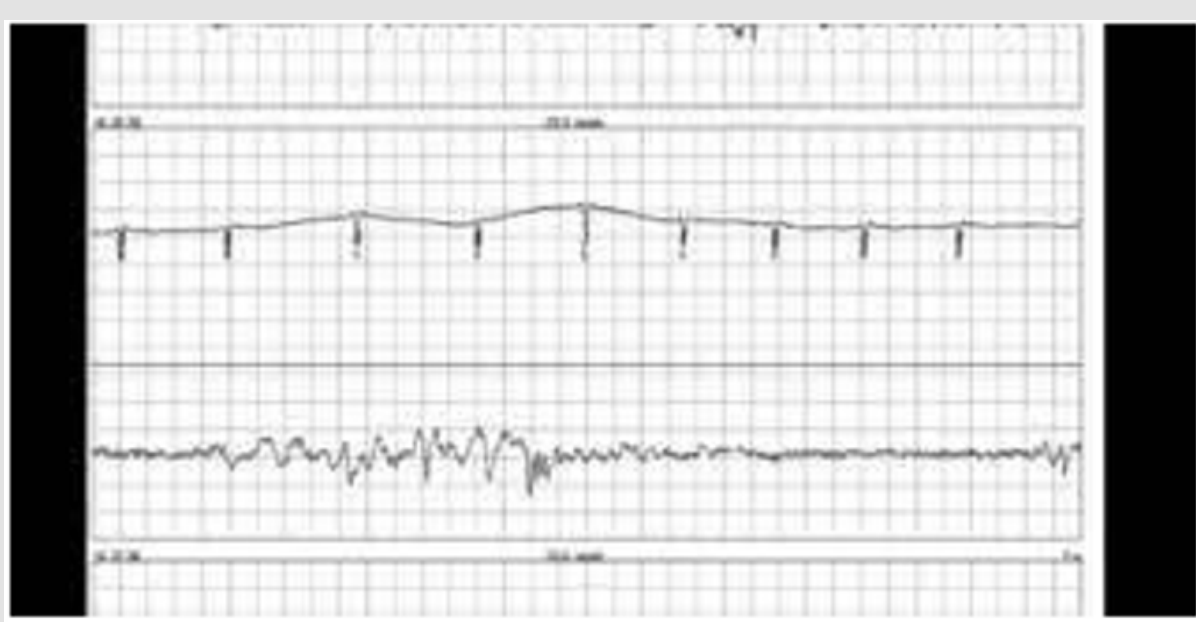

Figure 3.

\section{Complaints of Pain at the Device Site, Without Physical Findings}

Our service has experienced complaints from patients who had the device inserted at outside facilities and transferred care to our service. We have also received complaints from patients who had the device inserted at our facility with no obvious cause of the complaint. The complaint which is most common is one of pain at the insertion site out of proportion to any physical findings. The exam has shown to be normal in these cases and involves no redness, no streaking and no discharge from the site. Our usual work up includes discussion of specific complaints to pinpoint related events, signs and symptoms or underlying conditions which may affect the device site healing and any abnormal outcomes. Once the history and physical details are thoroughly discussed and examination will then be done. With the examination, often no offending findings are noted and there has been no erythema, rash, no swelling and no leakage.
We then move forward with a chest x-ray which often shows no culprit findings for the individual out of the anticipated level of pain for such a minor procedure. We often first recommend nonsteroidal anti-inflammatory medications such as ibuprofen. Our patients then make it clear that Tylenol and or Ibuprofen have done little to help with the pain at the insertion site. We have written for short term narcotics such as Vicodin for one week and this has helped with some of our patients. The Vicodin has solved the pain and irritation complaints at the site in approximately half of those who complain of this sensation at the loop recorder site. Of note, we don not continue prescribing the pain pills. We have also offered comfort measures including heat and cold to the area with mixed results. Ultimately there are less than $1 \%$ of our patients who continue to complain of pain or irritation at the device site longer than the first 2-3 weeks. However, we have experienced very interesting cases and we have investigated other possible sources of their pain. 
Our facility has evaluated three patients with almost identical complaints of a burning and gnawing sensation at the device site which caused "9-10/10" pain at the device site, despite Tylenol NSAIDs, and or Vicodin. These complaints continued for several weeks and were still present 3-4 months post insertion. Physical examinations were completely negative, chest $\mathrm{x}$-rays showed no change in position of the device and no migration of the device. Two of the patients received their device implants at outside institutions, one patient elected the removal of the device and one elected to do nothing and slowly over one year had resolving pain to the area. One patient started gabapentin with a resulting pain level down to " $2 / 10$ ". Specific care in insertion technique has resulted in avoiding insertion in areas of large amounts of subcutaneous fat. Selection of an ideal area for placement of the ILR is essential and some patients are not ideal candidates for loop recorders. Publications involving cases of migration of the device note the subcutaneous insertion technique and the nature in which the LINQ device is inserted lead to higher rates of migration and extrusion of the device without infection.

In a retrospective study of 85 patients who had undergone a LINQ insertion there was a $4.7 \%$ rate of spontaneous extrusion of the device within 7-24 days post insertion [22]. In our process of investigating possible sources of pain and local skin irritation from the loop recorder we have found cases of twiddler's syndrome. This syndrome involves the patient continually manipulating the site and subconsciously turns and rotates the ILR under the skin tissue. A clue that twiddler's syndrome may be present is continual changes in the upright versus downward QRS and obvious changes in axis of the waveform while monitoring the ongoing ECG tracings. The flipping of the QRS position in the home monitoring which can be seen within short time frames of each ECG tracing is a definite clue of possible twiddlers syndrome. This may result in local pain at the device site.

Another speculated source of possible change in position of the ILR is related tool which deploys the subcutaneous device. The tool provides an initial relatively wide opening in the skin, in comparison to the small profile of the LINQ device. The closure utilizing glue and approximating the margins is very successful in most insertions. There have been a few cases in which we have noticed changes in our ECG tracings and suspected movement of the device. We have brought the patient in for a physical examination and have noted the pocket site is rather larger in comparison to the LINQ device. We have then extracted and re-implanted out of the exact previous region, with very good success.

In our practice we have seen minimal LINQ ILR complications. The reference cases have caused us to be cautious in not delivering the device too deeply into the subcutaneous fat. We remain diligent in delivering the device to the recommended $8 \mathrm{~mm}$ depth. We have been cautious to meet with patients in person and to directly assess skin changes for possible extrusion or local rejection of the device. We continue to question cases of intense or prolonged pain with no local evidence of hematoma, no skin changes, and no radiographic evidence of migration. Some of these cases have resolved over several weeks with the use of analgesics and conservative local care with ice and pressure to the region. Others have required extraction of the device with no specific evidence of physical changes and intolerable pain to the area.

These cases both involved loop recorders which migrated from the original implant site and local skin changes and erosion at the insertion site. In our practice we have seen limited but similar cases involving one either potential migration of an ILR which was corrected shortly post insertion and one with device site pain requiring weeks of evaluation with conservative measures versus device explant. This required continued observation of these case scenarios to assure no late occurrence migration of the device. Our conclusions on an observational basis alone include the ILR use, and insertion continues to have a low side effect profile, but one in which the provider should be diligent to recognize and treat potential complications.

1. We have paid close attention to possible side effects of insertion of ILRs and would caution other providers to:

2. Take each post procedure complaint seriously.

3. Be aware that without physical findings or radiologic findings initially, that over time the device may migrate elsewhere or surface and extrude at the insertion site.

4. Remember to fully investigate post-operative complaints thoroughly which may entail repeat follow-up visits over several weeks.

5. Be cautious with the initial implants to follow the recommendations from Medtronic LINQ ILR package insert or other company recommendations and be cautious to avoid too shallow or too deep insertion of the ILR.

6. Be aware of the recipient's underlying health conditions and know that multiple disease states make a patient more susceptible to ILR site and migration complications postoperatively.

7. Manage pain with analgesics, ibuprofen and or Tylenol, and further investigate if pain is not decreasing. Suspect further complications or side effects if the pain is escalating. Remain diligent in follow up and suspect further complications if pain is not resolving.

8. Be aware of previous ILR side effect and cases in publication, to help guide the work up in patients who have post ILR complaints. 


\section{References}

1. Maines M, Angheben C, Cantanzariti D, Piffer L, Del Greco M, et al. (2018) Clinical impact, safety, and accuracy of the remotely monitored implantable loop recorder Medtronic Reveal LINQ. EP Europace 20(6): 1050-1057.

2. Beinhart S, Yang E (2020) Implantable loop recorder-Insertable cardiac monitor.

3. Dar T, Yarlagadda B, Gopinathannair R, Lakkireddy D (2018) Current Advances in wearable health technology: A review. EP Digest 18(3).

4. (2014) Zio patch helps diagnose heart conditions more accurately. ABC News.

5. Murbarik A, Iqbal A (2020) Holter monitor, Oakhill Hospital, Brookvile, Florida.

6. (2020) Medtronic. Indications, safety, and warnings for cardiac diagnostics and monitoring systems.

7. Bisignani A, Bonis S, Mancuso L, Ceravolo G, Bisignani G (2019) Implantable loop recorder in clinical practice. Journal of Arrhythmia 35(1): 25-32.

8. (2021) Implantable Cardiac monitors market expected to Rise by a CAGR of $4.4 \%$ by 2025 .

9. Oral H (2021) Estimate of yearly ILR insertions, University of Michigan Electrophysiology Department.

10. Sanders P, Piorkowski C, Kragten J, Goode G, Satish R (2019) Safety of Inhospital insertable cardiac monitor procedures performed outside the traditional setting: results of the Reveal LINQ in office 2 international study. BMC Cardiovascular disorders 19(1): 1-9.

11. Vilcant V, Kousa O, Hai O (2020) Injectable loop recorders-Stat pearlsNCBI Bookshelf.

12. (2020) Electrophysiology Protocol. Key elements of placement of ILRs. University of Michigan Hospital- Electrophysiology Department.

\section{ISSN: 2574-1241}

DOI: 10.26717/BJSTR.2021.36.005887

Kathleen Fasing. Biomed J Sci \& Tech Res

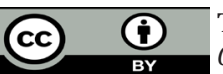

This work is licensed under Creative Commons Attribution 4.0 License

Submission Link: https://biomedres.us/submit-manuscript.php
13. Hasnie A, Hasnie A, Assaly R (2019) The case of the migrating loop Recorder. JACC Journals 1(2): 156-160.

14. Preminger M, Musat D, Sichrovsky T, Bhatt A, Mittal S (2017) Migration of an implantable loop recorder into the pleural space. Heartrhythm Case Reports 3(11): 539-541.

15. Porcoro C (2017) Implantable loop recorder leads to chronic pain. Expert Institute.

16. Syal S, Mwansa V, Chhabra L (2018) Unexpected silicone breast Implantation of insertable loop recorder. Methodist Debakey Cardiovascular Journal 14(2): e4-e6.

17. Drew D, Borici Mazi R, Baramchuk A (2015) Implantable loop recorder hypersensitivity. The Journal of innovations in cardiac rhythm management 6(8): 2109-2111.

18. Gunda S, Reddy Y, Pillarisetti J, Koripalli S, Courtney J, et al. (2015) Initial real-world experience with a novel insertable Reveal LINQ Medtronic compared to the conventional Reveal XT-Medtronic Implantable loop recorder at a tertiary care center- Points to Ponder. International Journal of Cardiology 191: 58-63.

19. Rogers J, Sanders P, Piorkowski C, Schail R, Anand R, et al. (2016) In office Insertion of a miniaturized insertable cardiac monitor: Results from the reveal LINQ In-office 2 randomized study. Heart Rhythm 14(2): 218-224.

20. Kipp R, Young N, Barnett A, Kopp D, Leal A, et al. (2017) Injectable loop recorder Implantation in an ambulatory setting by advanced practice providers: Analysis of outcomes. Pacing and Clinical Electrophysiology 40(9): 982-985.

21. Diederichsen S, Haugan K, Hojberg S. Holst A, Kober L, et al. (2017) Complications after implantation of a new-generation insertable cardiac monitor: Results from the LOOP study, International Journal of Cardiology 241: 229-234.

22.Zakhar J, Blount T, Gehi A, Ferns S (2020) Un-LINQed: Spontaneous Extrusion of newer generation implantable loop recorders. Indian pacing and electrophysiology journal 20(5): 189-192.

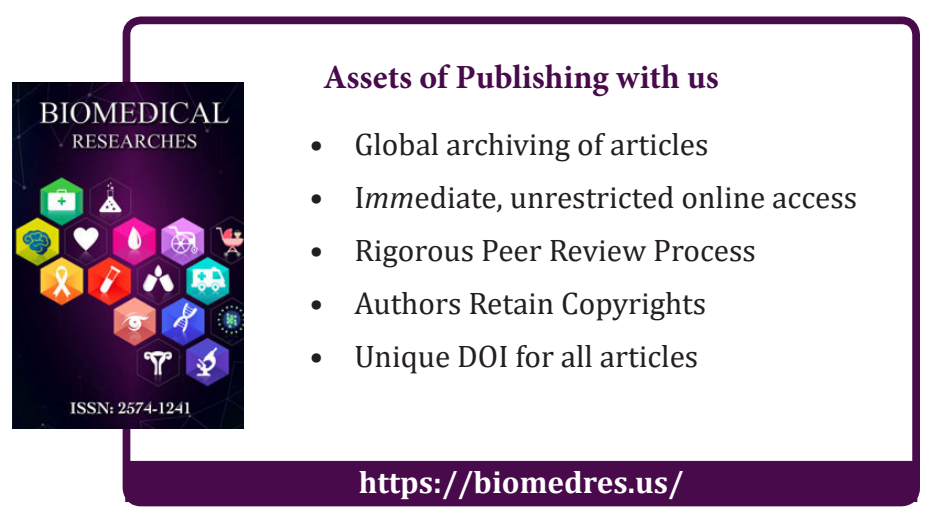

